\title{
Les processus d'urbanisation touristique sur la Costa Brava
} Joan Cals, Juli Esteban, Carles Teixidor

\section{Citer ce document / Cite this document :}

Cals Joan, Esteban Juli, Teixidor Carles. Les processus d'urbanisation touristique sur la Costa Brava. In: Revue géographique des Pyrénées et du Sud-Ouest, tome 48, fascicule 2, 1977. Barcelone et la Catalogne. pp. 199-208;

doi : 10.3406/rgpso.1977.3508

http://www.persee.fr/doc/rgpso_0035-3221_1977_num_48_2_3508

Document généré le 06/06/2016 
El desenvolupament del turisme a la Costa Brava i el fenômen d'urbanització que suposA. Descoberta pel turisma els anys 1930, la Costa Brava no ha estât explotada sistemàticament fins l'any 1955 sigui per satisfer el turisme internacional (clients individuals o grans masses manipulades per les empresses comerciáis) sigui per a crear residencies secundàries cercades per la burgesia barcelonina. En esdevenir el sol una riqueza especulativa l'economia regional ha quedat dominada pel mercat turistic. Pero aqueste transformado que no esta planificada a escala regional ha dut a malgastar recursos : els autors ho demqstren precisament en relació amb els plans parcials de vivendes en estudi que podrien aplegar mes de 500000 habitants, mes que la població de la provincia de Girona. L'anàlisis s'esten a una escala superior per recordar que les vivendes turístiques suposen un ordre urbà sensa implicar la créació de ciutats.

\begin{abstract}
The Costa Brava : the urbanization process induced by the growth of tourism. - Tourism appeared on the Costa Brava during the early Thirties but its systematic development was boosted only after 1955 . It consists in two main markets : the international one, with individual visits or properties and the vast masses manipulated by tour operators, and the regional market of the Barcelona bourgeoisie looking for holiday accomodations. This created a large speculation on land, and the whole regional economy is dominated, nowadays by tourism. Unfortunately this radical change was not planned at the region's level and it wastes a lot of space and ressources. It is stressed that the touristic compounds individually prepared in this area could accomodate half a million people, i.e. more than the provincial population of Gerona. The analysis is afterwards processed at a larger scale and demonstrates that such touristic compounds are an expression of urban principles but that they don't create consistent towns.
\end{abstract}

\title{
Résumé
}

El desenvolupament del turisme a la Costa Brava i el fenômen d'urbanització que suposA. Descoberta pel turisma els anys 1930, la Costa Brava no ha estât explotada sistemàticament fins l'any 1955 sigui per satisfer el turisme internacional (clients individuals o grans masses manipulades per les empresses comerciáis) sigui per a crear residencies secundàries cercades per la burgesia barcelonina. En esdevenir el sol una riqueza especulativa l'economia regional ha quedat dominada pel mercat turistic. Pero aqueste transformado que no esta planificada a escala regional ha dut a malgastar recursos : els autors ho demqstren precisament en relació amb els plans parcials de vivendes en estudi que podrien aplegar mes de 500000 habitants, mes que la població de la provincia de Girona. L'anàlisis s'esten a una escala superior per recordar que les vivendes turístiques suposen un ordre urbà sensa implicar la créació de ciutats.

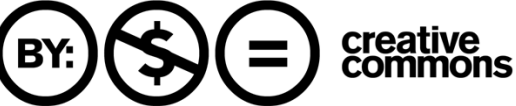




\title{
Les processus d'urbanisation touristique sur la Costa Brava
}

\author{
par Joan Cals *, Juli Esteban ** et Carles Teixidor **
}

\begin{abstract}
Les transformations urbanistiques de la Costa Brava, depuis sa conversion en une zone touristique, remontent pour sa partie méridionale au moins, à la fin des années 1950. L'explication essentielle des transformations tient du tourisme d'accueil, résidences secondaires comprises. Cette étude est centrée sur les lotissements, urbanizaciones, au travers desquels s'est réalisé un processus important de production et de consommation individualisée de l'espace; mais elle ne se désintéresse pas d'une vision globale du processus d'occupation du territoire...
\end{abstract}

\section{Tourisme et économie sur la Costa Brava}

La Costa Brava fut découverte par le tourisme international vers 1928-1930, avec l'arrivée des premiers Allemands à Tossa, et la création de S'Agaró (1). En 1936, la majeure partie du littoral restait en marge du marché touristique dont les flux ne reprirent que 20 ans plus tard, après la guerre civile et la guerre mondiale.

Le tableau I souligne les traits remarquables de l'évolution de la capacité hôtelière : forte croissance jusqu'à 1968, récession à partir de 1972, augmentation des dimensions moyennes des établissements (la baisse du nombre d'établissements s'accompagne d'une croissance de la capacité totale de logement), part considérable de Lloret de Mar, qui comptait, en 1975, 203 hôtels et 15528 chambres.

Il est important de retenir ces caractéristiques du comportement du sous-secteur le plus représentatif de l'industrie touristique, sans qu'on puisse les extrapoler à l'ensemble de l'offre en logement pour laquelle il n'y a pas de statistiques fiables; on peut dire de l'évolution depuis 1972 qu'elle est plus « positive »: la construction d'appartements, bungalows, etc., à usage touristique continue après

(*) Professeur d'Economic à l'Université autonome de Barcelone.

(**) Architecte-urbaniste, Corporació Metropolitana de Barcelona et Equip Taller d'Architectura i Urbanisme.

$(* * *)$ Architecte-urbaniste, Institut Provincial Urbanisme de Barcelone et Equip Plancjament Urbanists Associats.

(1) Y. BarbazA, Le paysage humain de la Costa Brava. Paris, 1966, A. Colin. 
TABI.EAU 1

Evol.ution du poteNtiel hôtelier de l.a Costa Brava

\begin{tabular}{|c|c|c|c|}
\hline Année & $\begin{array}{c}\text { Nombre } \\
\text { d'étabiissements }\end{array}$ & $\begin{array}{c}\text { Nombre } \\
\text { de chambres }\end{array}$ & $\begin{array}{c}\text { Chambres } \\
\text { établissement }\end{array}$ \\
\cline { 2 - 4 } 1956 & 255 & 5105 & 20 \\
1960 & 532 & 11580 & 21,7 \\
1964 & 643 & 19310 & 30 \\
1968 & 721 & 23384 & 32,4 \\
1972 & 756 & 32007 & 42,3 \\
1974 & 726 & 32223 & 47,2 \\
1975 & 737 & 34782 & 47,2 \\
\hline
\end{tabular}

la stagnation de 1974-75, comme continue la construction de résidences secondaires dont les propriètaires viennent surtout de l'aire métropolitaine de Barcelone.

En réalité, le tableau I reflète la crise profonde qui affecte le secteur touristique (2). Non pas crise de conjoncture, mais phénomène plus profond - accentué sans doute par la récession de l'économie capitaliste -, découlant du modèle même de développement touristique adopté par l'Administration de Madrid. C'est la conséquence inévitable d'une politique touristique de grandeur, de l'usage que cette politique a fait du secteur touristique, des critères spéculatifs qui l'on orienté et de son propre manque de rationalité, tant en termes micro que macro-économiques (3).

Si, d'un point de vue purement spatial on peut distinguer trois secteurs sur la Costa Brava (pyrénéen, de la frontière à Rosas; entre Rosas et Bagur, incluant une grande partie de la plaine de l'Ampurdan, avec la discontinuité du massif de Montgrí; méridional, de Bagur à Blanes), on peut évoquer, dans une optique touristique, un double marché : dans quelques centres, Lloret, l'Estartit, Tossa, Rosas et Blanes, etc., le tourisme dominant est celui des " tour operators », en croissance rapide à partir de 1965; ailleurs, au contraire, la clientèle touristique continue à être, fondamentalement, individuelle ou particulière.

L'irruption du tourisme a provoqué des changements importants dans la répartition des ressources et dans la structure économique du littoral (4). Il est intéressant de détacher spécialement ici ce qui

(2) A. Gibert, El crack turistico de la Costa Brava. Gérone, 1972, Cambra de Comerç i Industria.

(3) J. Cals, Turismo y politica turistica en España : una aproximaçion. Barcelone, 1974, Ariel.

(4) Mais l'effet reste inégal en fonction, surtout, du degré d'industrialisation (industries du liège en général) de chaque zone. Cf. P. Campistol et E. Lluch, Les consequencies comarcals del turisme a Costa Brava. Barcelone, 1972, Banca Catalana. 
se réfère au sol, une ressource naturelle revalorisée soudainement, dans la mesure où elle passe, de façon irréversible, d'un usage essentiellement agricole et forestier à des usages de type touristicorésidentiel. La capitalisation de ces rentes par les anciens propriétaires, enrichis par la vente, se traduit dans un premier temps par des investissements dans l'hôtellerie ou le commerce en liaison avec le tourisme et ces investissements déterminent un développement économique large et profond.

Progressivement, l'économie se calque sur le secteur touristique et autres secteurs de demande induite, avec lesquels dépendance et vulnérabilité augmentent largement. Le secteur de la construction prend une importance croissante; pour maintenir son niveau d'activité et en l'absence d'alternatives possibles il est nécessaire d'accroître la monoculture, ce qui s'accomplit au prix de coûts sociaux extraordinairement élevés.

L'absence d'une politique économique en matière d'équilibre régional, les privilèges octroyés à la spéculation immobilière comme mécanisme d'accumulation et la crise chronique des activités agraires sont, probablement, les causes immédiates qui ont empêché qu'un excédent économique volumineux, fruit d'une conjoncture exceptionnellement favorable sous bien des aspects, puisse servir à diversifier la structure économique de la côte : un pas indispensable pour corriger sa vulnérabilité et obtenir un système qui assure une croissance soutenue sans avoir à recourir à «l'auto-destruction ».

\section{Le cadre institutionnel de l'urbanisme}

Différents agents interviennent dans le procès d'occupation de l'espace : promoteurs privés, propriétaires du sol, constructeurs, et administration publique, à laquelle nous accorderons une brève attention; on constate d'abord, à son propos, une grande dispersion des compétences et un manque de coordination dans leurs interventions, ce qui a eu des conséquences négatives.

$\mathrm{Ni}$ la Costa Brava dans son ensemble, ni ses différents secteurs n'ont compté avec une politique cohérente d'aménagement du territoire. L'unique tentative réalisée, il y a dix ans, est due au Ministère de l'Information et du Tourisme qui a étudié la possibilité de déclarer la Costa Brava "Zone d'Intérêt touristique national ", mais abandonna par la suite le projet.

Laissant de côté les plans, presque toujours privés, des lotissements, la planification de l'urbanisme a été exercée par les municipalités, avec un contrôle bureaucratique extrémement pirmissif de la Délégation provinciale du Ministère du Logement. Cette planification municipale, réalisée à travers les «Plan géné- 
raux d'Aménagement urbain ", répond à une vision fragmentée de la réalité qui favorise la spéculation sur le sol. Cela paraît finalement logique, lorsque l'on connaît le manque de représentativité des hommes publics et les liens directs ou indirects d'un grand nombre de maires et de notables avec les intérêts des propriétaires ou des sociétés immobilières.

Quant aux interventions du secteur public, la Costa Brava est un exemple clair de "l'atrophie du capital social ", un des traits les plus caractéristiques du modèle du capitalisme sauvage utilisé par le franquisme qui, dans le cas de la Catalogne, s'accentue comme conséquence du faible taux de retour des apports financiers versés à l'Administration d'Etat. Indubitablement, le littoral géronais a fait les frais de graves discriminations par rapport à d'autres zones touristiques : le Ministère des Travaux publics a accordé de 1969 à 1975, 1838 millions de pesetas à la province de Gérone dans le domaine des infrastructures (et plus spécialement des voies de communication) contre 9701 millions au secteur Malaga-Costa del Sol (5).

\section{La consommation individualisée de l'espace : les lotissements}

L'occupation de l'espace sur la Costa Brava se manifeste à travers la croissance des noyaux urbains traditionnels et par la promotion des lotissements, ce dernier phénomène étant le plus important. Les mécanismes évoqués présentent, évidemment, des différences; mais la structuration de l'implantation humaine sur le territoire dépend tant de l'un que de l'autre; ce serait, donc, une erreur que de poser les problèmes séparément, tout comme de centrer l'étude sur les déficits et problèmes du noyau traditionnel, en ignorant les lotissements, ce qui signifierait qu'on les considère comme un fait autonome et qu'on leur dénie toute incidence en dehors de leur propre espace.

Dans les communes côtières du Haut-Ampurdan, du Bas-Ampurdan, et de la Selva, douze plans partiels visant 215 hectares étaient déposés à la fin de 1960, 134 plans pour un total de 3582 hectares à la fin de 1965 et 287 plans sous 7312 hectares à la fin de 1974 (d'après le Collège des Architectes de Gérone).

La réalisation totale des prévisions contenues dans les plans (ce qui est loin de se produire) supposerait une occupation poussée de l'espace, pour accueillir une population de quelques 500000 personnes; un chiffre qui tout en étant élevé en termes absolus (la

(5) J.M. Muntañer Pascual, El plan de accesos a la Costa Brava : encens per a beneits, Presencia, Gérone, août 1975. 
population résidant habituellement dans la province de Gérone s'élevait en 1970 à 414000 personnes) reste réduit par rapport à la superficie.

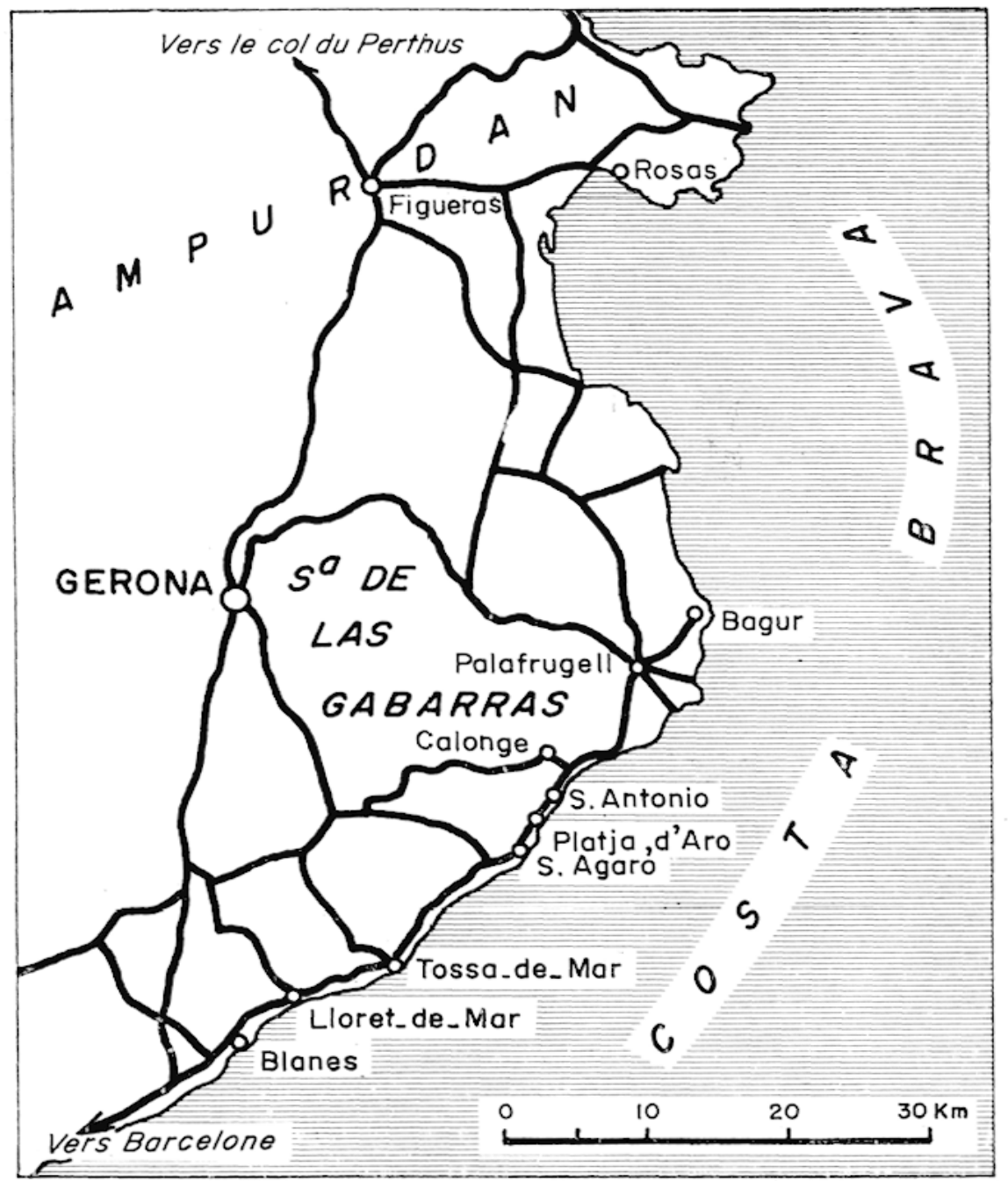

Fig. 1

Desserte routière de la Costa Brava

L'extraordinaire excédent d'offres que traduisent ces chiffres ressort plus nettement si l'on considère les volumes atteints par les offres concurrentielles, comme le sont maintenant les 475 lotissements (19 550 hectares) en cours de réalisation dans le cadre de l'aire métropolitaine de Barcelone, et les 4250 hectares de "Plans Partiels " approuvés dans la province de Tarragone (6).

(6) Chiffres extraits de l'étude sur les résidences sccondaires, en 1974-1975 (Aire métropolitaine de Barcelone). 


\section{Caractéristiques du lotissement-type.}

Pour parler de lotissements au sens large, il faut que les interventions aient pour base territoriale des unités de propriété rurale ou de fragments d'une propriété (c'est-à-dire que s'urbanise un secteur qui ne soit pas divisé entre différents propriétaires) et que le projet de construction repose sur l'utilisation du modèle de parcellaire réservé aux logements « unifamiliaux », connu vulgairement sous le nom de "cité-jardin " par dégénérescence de ce concept qui, historiquement, a signifié plus qu'un modèle de lotissement.

Le fait que les lotissements se réalisent à terme dans le cadre d'une propriété unique apporte un avantage fondamental : l'indépendance au niveau de l'intervention.

L'adoption de la cité-jardin comme modèle d'aménagement ne dérive pas seulement de la demande, opportunément stimulée, mais aussi de raisons profondes. Ainsi du rapport entre lotissement et infrastructures : on peut donner, avec un investissement minimum (ouverture et nivellement des routes), accès à la parcelle, ce qui permet à celle-ci d'être vendue immédiatement; le reste des travaux d'urbanisation peut être différé et permettre de majorer le prix pour les autres acheteurs.

- La réalisation du lotissement peut se diviser en phases, ayant pour objet d'urbaniser différentes parties de l'aire programmée, en les ajustant aux possibilités financières du promoteur.

- Les carences de l'urbanisation (asphaltage défectueux, défaut de revêtement pour les trottoirs, etc.) sont mieux supportés par la population; les faibles densités rendent moins graves certaines carences.

- La gestion indépendante de la construction est possible, ce qui satisfait l'individualisme de l'acheteur; cela rend compatible des objectifs d'achat différents, comme le simple investissement dans les terrains, et surtout, cela libère le promoteur d'une obligation qui exigerait une plus grande capacité financière et un risque plus élevé dans l'opération.

Vérifiée la logique des caractéristiques qui définissent le lotissement, apparaissent ses conséquences immédiates. L'aire concernée par les lotissements correspond rarement à la morphologie du territoire; ainsi disparaît la logique de la "position" (7). Le processus de succession temporelle et de "localisation " dans l'espace des actions d'urbanisation, ne coïncide pas avec les normes traditionnelles ou planifiées de la croissance urbaine. La dispersion des

(7) La " position » est employée dans le sens de relation entre l'aire soumise à urbanisation avec l'espace spécifique où elle s'implante. Il s'agit d'une relation fondamentalement morphologique et nous la distinguons ainsi de la "localisation " ressentie comme un processus soumis à un plus grand nombre de variables. 
interventions dans le temps et dans l'espace - s'appuie de manière unique et indispensable sur le réseau routier existant, entraînant sa déconnexion. D'un autre côté, la liberté d'implantation multiplie gravement l'agression du milieu naturel.

Quant à l'ineffable "cité-jardin ", qui sert de modèle d'aménagement pour tous les lotissements, on peut affirmer qu'elle entraîne un gaspillage important de terrain, en fonction du nombre d'usagers qui bénéficieront de l'intervention, et que les coûts d'urbanisation par habitant sont, logiquement, plus élevés, tant en raison des normes (par exemple mètres-linéaires de rue par habitant) que par la dispersion des interventions, qui rend impossible un usage rationnel des infrastructures générales (assainissement, alimentation en eau, etc.). Ces coûts seraient aussi très importants si l'on considérait l'entretien des services (éclairage, collecte d'ordures, etc.); enfin, ils rendent très difficiles les finances municipales.

Le peu de population que peuvent accueillir les lotissements en raison de leur taille empêche la mise en place d'une gamme complète de services pour les habitants eux-mêmes, créant une situation de dépendance vis-à-vis des centres urbains, en contradiction avec les prétentions d'autonomie et d'indépendance que sous-entendent ces actions.

\section{Effets des lotissements sur la structuration de l'espace.}

Avant l'irruption du tourisme, la Costa Brava était une aire structurée par la localisation, avec une logique géographique facilement compréhensible, d'une série de noyaux urbains de fonction et d'importance inégales, reliés par un réseau de routes leur permettant de communiquer entre eux et avec d'autres centres.

L'urbanisation dispersée altère le système de noyaux urbains existants. Le terrain qu'occupent les lotissements n'est guère plus que celui que l'on pourrait considérer comme ayant des expectatives urbaines en raison de l'expansion du noyau traditionnel. De même se pose le problème de la correspondance avec le terrain que le "Plan général d'Aménagement urbain » de la Commune inclut dans ses prévisions d'urbanisation.

La logique de production des lotissements, de la même manière qu'elle n'a rien à voir avec les aptitudes morphologiques du territoire, n'est pas davantage reliée aux prévisions de croissance. S'urbanisent les propriétés de ceux qui le décident, directement ou à la suite d'une vente; ne s'urbanisent pas celles dont les propriétaires préfèrent attendre.

La localisation des initiatives en matière d'urbanisation est, par là-même, totalement imprévisible et l'intervention acquerra une signification bien différente si elle est proche du noyau urbain de la Commune. Los Pinares, les Teules, Mas Ambros, etc., n'appartiennent pas à l'idée que l'on a de Lloret, Platja d'Aro, Calonge, 
etc.; ce sont des secteurs développés à l'intérieur du cadre administratif de ces communes, mais dans la mesure où elles ne peuvent être assimilées à un agrandissement des centres urbains, et qu'elles répondent à une idée d'autonomie, elles se présentent comme des options différentes du noyau traditionnel. On pourrait dire qu'il s'établit une certaine concurrence entre les deux modèles urbains.

Les lotissements donnent lieu à une remise en question du système urbain existant. Les causes immédiates de ce parasitisme se trouvent dans la propre organisation morphologique de l'espacc et dans la position qu'ils adoptent, en liaison avec l'infrastructure générale des voies qui desservent le territoire. L'organisation de l'espace réalisée avec des critères dérivant de la taille, de la forme et de la topographie de la propriété rurale, donne lieu à une organisation refermée sur elle-même, avec autant de culs-de-sac qui conviennent à la parcellisation totale du territoire, sans interférer avec les expectatives des propriétaires voisins. Cet aménagement a une relation unique et inéluctable avec le système urbain existant, grâce à la route la plus proche, celle qui permet d'accéder au lotissement. Il est facile de comprendre que, avec cette mécanique, la juxtaposition d'espaces urbanisés à ce qui existe ne produira jamais une maille capable de se convertir en un support structurel de la nouvelle dimension urbaine que représente un territoire urbanisé dans sa majorité. Une telle responsabilité reste confiée de façon permanente aux voies de communication déjà existantes, qui se voient forcées ainsi à jouer un rôle d'accès et de communication pour lesquels elles n'avaient pas été prévues.

La dispersion morphologique et les voies de communication reliant les lotissements au noyau urbain empêchent, d'autre part, la possibilité d'un dédoublement progressif du centre de services de la commune, qui permettrait la mise en place d'un dispositif capable de desservir la nouvelle organisation résidentielle.

Les lotissements ne se contentent donc pas de ne pas contribuer à charpenter le territoire de la Costa Brava, mais ils le transforment de façon irréversible, hypothéquant une bonne part de ses espoirs. Le collapsus urbanistique que l'on peut prévoir, si se maintiennent les pratiques « d'urbanisation » avec la virulence des dernières années, marquera la limite à l'utilisation d'un espace qui pouvait et devait donner beaucoup plus.

\section{Lotissement et privatisation du territoire.}

La terre est ici presque toujours propriété privée. Toutefois le régime de propriété, dans le cadre rural, n'empêche pas, normalement, qu'elle que soit la destination du terrain, l'accès et la possibilité d'en jouir largement. Or, le changement que représente le passage du rural à l'urbain comporte une limitation des droits d'utili- 
sation du sol. Des terrains spécifiquement publics apparaissent (rues, places, zones vertes, etc.) et, en contrepartie, le domaine privé se renforce sur le reste du sol, converti en terrain à bâtir. Le lotissement est un des mécanismes de ce processus et cela se vérifie aussi bien pour les parcelles de 400 , de 2000 que de 10000 mètres carrés, ce qui disqualifie la thèse selon laquelle de grandes parcelles permettent le maintien du milieu rural.

Dans notre perspective, le processus " urbanitzador-generic » n'est pas un simple processus de privatisation du sol : il implique la polarisation des deux sphères, publiques et privées, dans lesquelles se développe l'activité humaine. Cela se traduit physiquement par une accentuation de l'aspect privé de certains espaces et, en échange, par la définition et la délimitation d'espaces d'utilisation spécifiquement publique, qui n'existent pas dans l'espace rural. Des proportions et des qualités de ces espaces, dépendra l'équilibre du nouveau milieu de vie urbaine.

Dans les lotissements dont nous venons de traiter, on prouve facilement que l'apport d'éléments publics, en contrepartie de la privatisation " à l'échelle urbaine » du sol, ne se produit pas réellement. L'objectif primordial du lotissement est la parcellisation, provoquant ainsi une hypertrophie qui porte préjudice aux autres composants du concept "generic d'urbanització " (services, édification, espace urbain, etc.). En conséquence, les espaces publics restent réduits aux rues conçues comme de simples voies d'accès aux parcelles, et à une série de bouts de terrains difficiles à lotir, en raison de leur position ou de leur topographie. Ces rues semi-privées, avec une ordonnance en labyrinthe, n'ont aucune utilité pour les citadins étrangers aux lotissements. Les périmètres d'action définissent des véritables aires privatisées, autant par référence à l'espace rural qu'à celui que l'on peut proprement dénommer urbain.

Tout cela reste très loin de l'idée initiale de cité jardin. Rappelons E. Howard, le premier théoricien de ce modèle urbanistique, et d'autres pionniers, tels Unwin ou Cebrià de Montoliu, et nous verrons que ce qu'ils proposaient était certainement une ville. Le lotissement n'est pas une troisième voie entre l'urbain et le rural; c'est plutôt un piège pour éviter ces composantes spécifiquement urbaines, rejetées non pour des questions de qualité, mais parce qu'elles pourraient donner lieu à des interférences dans la base commerciale de l'opération.

(8) Les parcelles de grande taille $\left(5\right.$ à $\left.10000 \mathrm{~m}^{2}\right)$ sont fréquentes dans les terrains situés en deuxième ligne de la côte, là où la demande n'est pas
encore aussi forte. Il s'agit toujours de terrain qualifié de rural dans le "Plan général " et avec ce type de parcelle on prétend éviter la rédaction d'un "Plan partiel", en supposant que les parcelles sont " proprićté rurale" ". Contre cette manipulation s'est manifestée dernièrement, dans les Gavarres en particulier, une forte réaction populaire.

(9) H.P. BARTh, Lineamenti de sociologie urbanistica. Padova, 19..., Marsilio. 
On peut dire, en résumé, que l'on urbanise - et parfois de façon correcte -, mais sans faire de ville. Nous voilà donc revenus, et pas par hasard, à ce que nous exposions précédemment, au sujet du manque de structuration en matière d'urbanisme sur la Costa Brava. Individualisme (dans les interventions) et privatisation (comme résultat) sont des concepts cohérents et tous deux contraires à la logique de formation d'une véritable ville, ainsi qu'à l'utilisation de l'espace en accord avec la nécessité collective.

Réscuŕ. - Découvert par le tourisme vers 1930, le littoral de la Costa Brava n'a commencé d'être exploité systématiquement qu'à partir de 1955 soit pour satisfaire le tourisme international (clientèle individuelle ou grandes masses manipulées par les entreprises commerciales) soit pour créer les résidences secondaires recherchées par la bourgeoisic de Barcelone. Le sol devenant une richesse spéculative, l'économie régionale est dominée par le marché touristique. Mais cette transformation n'est pas planifiée à l'échelle régionale et conduit au gaspillage; les auteurs le montrent notamment à propos des plans partiels des lotissements à l'étude qui pourraient accucillir plus de 500000 habitants, plus, que la population de la province de Gérone. L'analyse est poursuivie à grande échelle pour rappeler que ces lotissements touristiques relèvent de l'ordre urbain mais ne créent pas de villes.

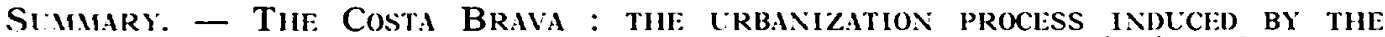
GROWTI OF TocRISM. - Tourism appeared on the costa Brava during the early Thirties but its systematic development was boosted only after 1955. It consists in two main markets : the international one, with individual visits or properties and the vast masses manipulated by tour operators, and the regional market of the Barcelona bourgeoisie looking for holiday accomodations. This created a large speculation on land, and the whole regional cconomy is dominated, nowadays by tourism. Unfortunately this radical change was not planned at the region's level and it wastes a lot of space and ressources. It is stressed that the touristic compounds individually prepared in this area could accomodate half a million people, i.e. more than the provincial population of Gerona. The analysis is afterwards processed at a larger scale and demonstrates that such touristic compounds are an expression of urban principles but that they clon't create consistent towns.

El dESENVOLLPAMENT DEL TLRISME A IA COSTA BRAVA I EL FENOMMEN d'URBANITZACIÓ QUE SUPOSA. - Descoberta pel turisma cls anys 1930, la Costa Brava no ha estat explotada sistemàticament fins l'any 1955 sigui per satisfer el turisme internacional (clients individuals o grans masses manipulades per les empresses comercials) sigui per a crear residències secundàries cercades per la burgesia barcelonina.

En esdevenir el sòl una riqueza especulativa l'economia regional ha quedat dominada pel mercat turistic. Pero aqueste transformació que no esta planificada a escala regional ha dut a malgastar recursos : els autors ho demostren precisament en relació amb els plans parcials de vivendes en esiudi que podrien aplegar més de 500000 habitants, més que la població de la província de Girona. L'anàlisis s'esten a una escala superior per recordar que les vivendes turístiques suposen un ordre urbà sensa implicar la créació de ciutats. 